\title{
GREEK WORDS
}

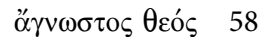

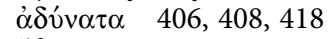

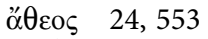

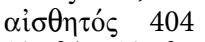

$\dot{\alpha} \lambda \eta \theta \hat{n} \varsigma, \dot{\alpha} \lambda \eta \theta$ vós $\quad 446,450$

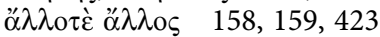

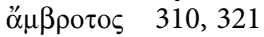

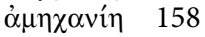

óv $\alpha \xi \quad 136$

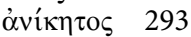

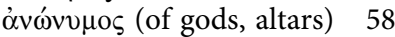

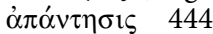

$\dot{\alpha} \pi \hat{i} \theta \alpha v \alpha \quad 406,418$

$\dot{\alpha} \pi \mathrm{o} \delta \eta \mu \dot{i}_{\alpha}(\mathrm{l}) \quad 90 \mathrm{f}$.

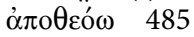

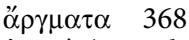

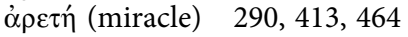

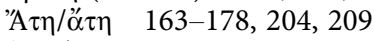

$\dot{\alpha} \tau \mu \mu^{\prime} \alpha \quad 117$

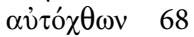

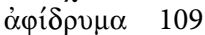

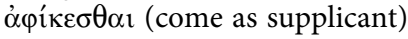

410

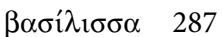

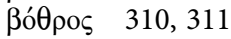

$\beta \omega \mu$ ós 311

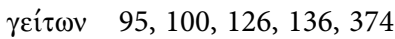

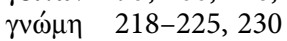

Soís 312, 367-369

$\delta \varepsilon ı \sigma ı \alpha i ́ \mu \omega v \quad 127$

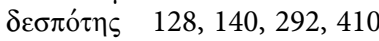

$\delta 1 \delta \alpha \sigma \kappa \alpha \lambda i ́ \alpha \quad 130$

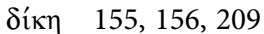

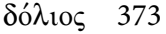

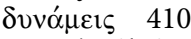

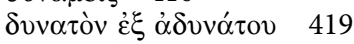

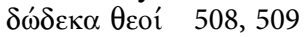

है $\gamma \chi \omega \rho(1)$ os $95,98,99,101,116$

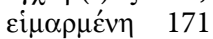

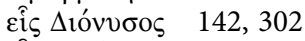

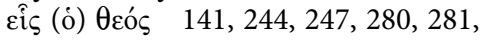
296-301

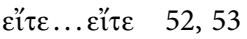

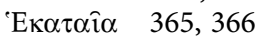

हैк $\pi \lambda \eta \xi_{1 \varsigma} \quad 478$

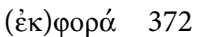

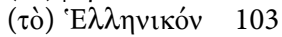

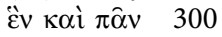

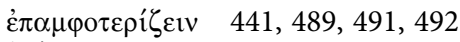

غ̇лท́кооऽ 449

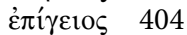

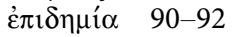

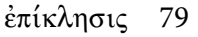

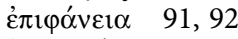

$\dot{\varepsilon} \pi \omega v 0 \mu i ́ \alpha \quad 77$

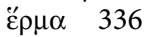

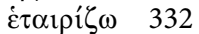

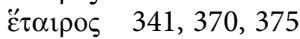

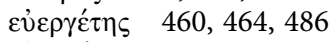

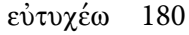

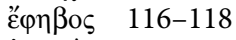

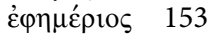

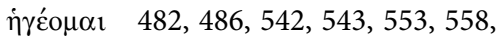
559

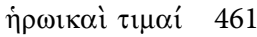

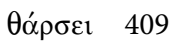

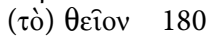

$\theta$ cîos 460

(oi) $\theta$ coí $\quad 268-278$

'E $\lambda \lambda$ ńvior 105

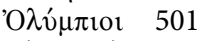

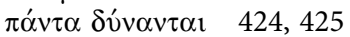

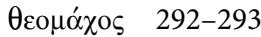

$\theta \varepsilon o \mu$ orpía 359

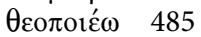

(o) $\theta \varepsilon o ́ \varsigma \quad 169,176-178,185,270-272$

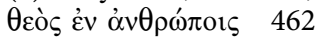

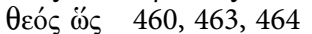

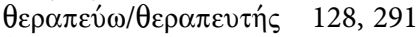

$\theta \varepsilon \hat{\omega}$, see: $\tau \grave{\omega} \theta \varepsilon \hat{\omega}$

$\theta v \lambda \hat{n} \mu \alpha \tau \alpha \quad 363$

ió $\mu \alpha \tau \alpha \quad 400,404-416$

i $\alpha \tau \rho \alpha \quad 416$

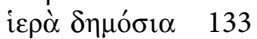

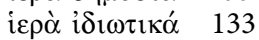

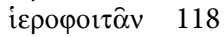

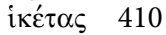

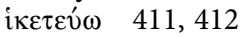

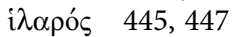

¡入 $\lambda \sigma \kappa \varepsilon ́ \sigma \theta \alpha \mathrm{r} \quad 44$ 


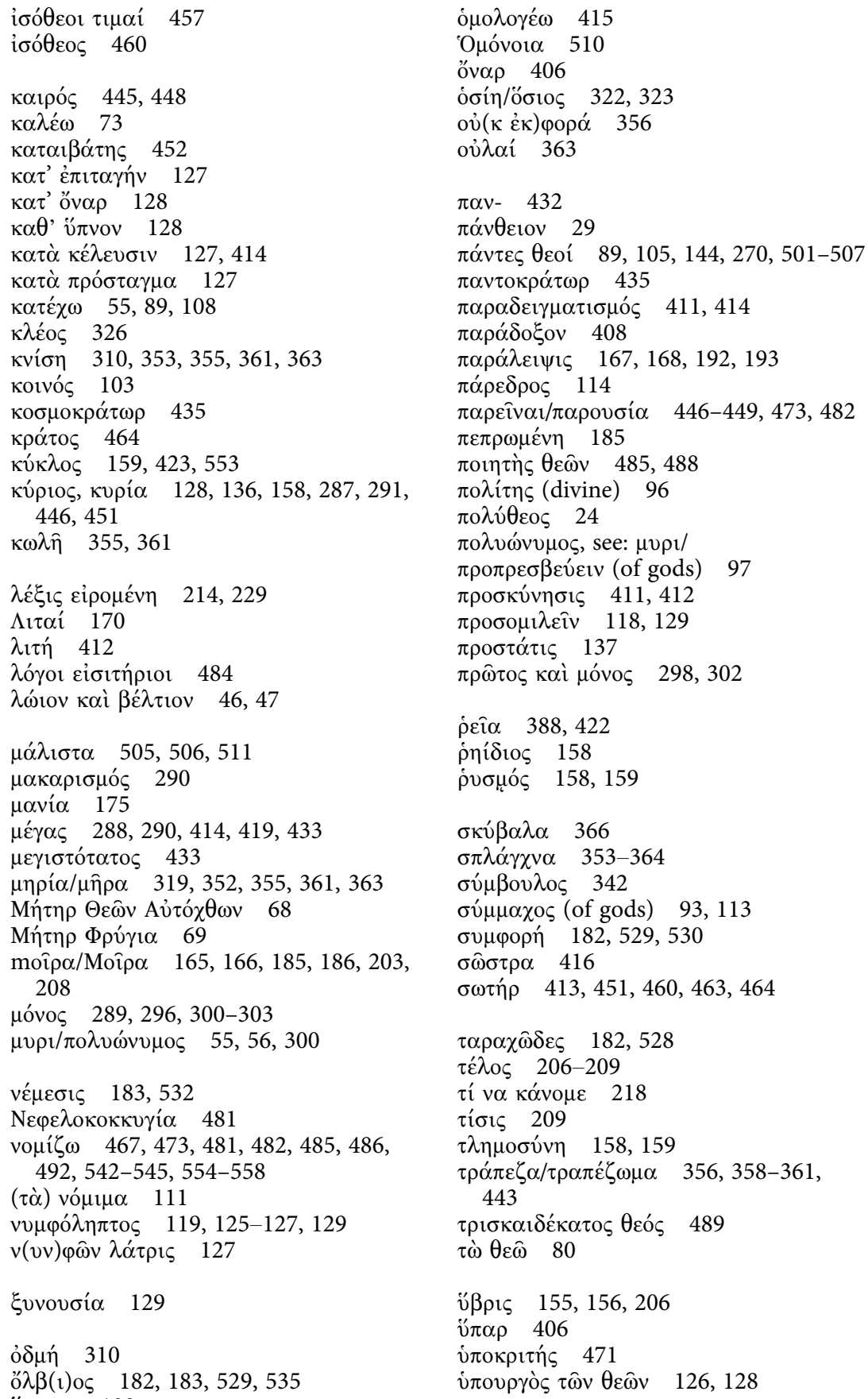


$\varphi \alpha ́ \sigma \mu \alpha \quad 402,404$

$\varphi 1 \lambda \alpha \nu \theta \rho \omega \pi$ ó $\alpha \tau \varepsilon \quad 318,341,402$

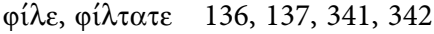

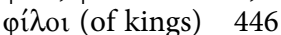

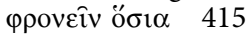

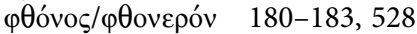

$\tau \varepsilon \kappa \alpha i ̀ ~ \tau \alpha \rho \alpha \chi \hat{\omega} \delta \varepsilon \varsigma \quad 528$

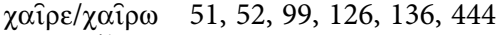

$\chi \rho \eta \mu \alpha \tau^{\prime} \zeta \omega$

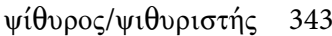

" $\Omega$ por 73

ஸ́ 473, 488, 493, 494

see also: as if

ITALIC/LATIN WORDS

frequentare templa 118

sive... sive 57

sive deus, sive dea 58
Tursa Serfia Serfer Martier 115

unus/una 289, 297, 300 